\title{
Hierarchical cascade model leading to 7 -th order initial value problem
}

\author{
${ }^{*}$ Ghazala Akram ${ }^{\dagger}$, Christian Beck ${ }^{\ddagger}$
}

\begin{abstract}
In turbulent flows, local velocity differences often obey a cascade-like hierarchical dynamics, in the sense that local velocity differences at a given scale $k$ are driven by deterministic and random forces from the next-higher scale $k-1$. Here we consider such a hierarchically coupled model with periodic boundary conditions, and show that it leads to an $N$-th order initial value problem, where $N$ is the number of cascade steps. We deal in detail with the case $N=7$ and introduce a non-polynomial spline method that solves the problem for arbitrary driving forces. Several examples of driving forces are considered, and estimates of the numerical precision of our method are given. We show how to optimize the numerical method to obtain a truncation error of order $O\left(h^{5}\right)$ rather than $O\left(h^{2}\right)$, where $h$ is the discretization step.
\end{abstract}

\section{Introduction}

Hierarchical dynamics arise quite commonly for complex systems that consist of many subdynamics that drive each other in a selfsimilar way. Often, the dynamics at a given scale $k$ couples to the dynamics at a higher scale $k-1$ in a simple way, and these types of problems can sometimes be dealt with in an analytic way. A typical example are cascade models in turbulence (see, e.g. 1, 2, 3, and references therein), which are a useful tool to characterize the selfsimilar features of turbulent flows at high Reynolds numbers.

In this paper we consider a very simple hierarchical model that can be physically interpreted as representing velocity differences at different scales that are driven by deterministic and stochastic forces from the next higher scale, in a medium with viscosity and a given driving force at the top scale. We show that the problem reduces to the solution of an initial value problem associated with a differential equation of order $N$, where $N$ is the number of cascade steps. As an example, we deal in detail with the case $N=7$, which corresponds to typical values of cascade sizes observed in turbulent flows. There are three standard approaches to solve initial value problems numerically, the finite difference method, the finite element method and the spline approximation methods. We introduce a non-polynomial

*Department of Mathematics, University of the Punjab, Lahore 54590, Pakistan. Email: toghazala2003@yahoo.com

${ }^{\dagger}$ School of Mathematical Sciences, Queen Mary, University of London, Mile End Road, London E1 4NS, UK. Email: g.akram@qmul.ac.uk

${ }^{\ddagger}$ School of Mathematical Sciences, Queen Mary, University of London, Mile End Road, London E1 4NS, UK. Email: c.beck@qmul.ac.uk 
spline method for the numerical solution of this initial value problem, and estimate the precision of our numerical treatment. Higher-order boundary value problems are effectively solved using non-polynomial spline methods [5, 6, 7, 8, 9]. It turns out there is an optimum choice of the interpolation parameters where our methods yields best possible results (5th order rather than 2 nd order in the discretization step). Some analytically solvable examples of driving forces are dealt with as examples, for illustration of our general method.

\section{Cascade model}

Let us consider a model of damped particles in a viscous medium that are driven by rapidly fluctuating forces. Suppose there are $N$ such particles. We denote the velocity of each particle as $y^{(k)}(t), k=1,2, \ldots, N$. A very simple, uncoupled model would be that the particles are damped by a linear friction force, which is proportional to velocity, and a rapidly fluctuating driving force $L^{(k)}(t)$ that is independent of velocity:

$$
\dot{y}^{(k)}=-\Gamma y^{(k)}+L^{(k)}(t)
$$

$\Gamma>0$ denotes the friction constant. If $L^{(k)}(t)$ is Gaussian white noise, then this model just leads to the Ornstein-Uhlenbeck process, performed independently by each particle [4].

Here, however, we want to modify this model to a more interesting interacting dynamics. First of all, we allow $L^{(k)}(t)$ to be any time-dependent driving force, and assume that it is differentiable. Next, we construct a coupled hierarchical model, by replacing $y^{(k)}$ on the right-hand side of the above equation by the nearest neighbour $y^{(k+1)}$. We may physically interprete $y^{(k)}$ as a local velocity difference in a turbulent flow at spatial scale $r=2^{-k}$. The physical interpretation is that the change of velocity due to friction forces at a given scale $k$ is proportional to the velocity at the next-smaller scale $k+1$. This reminds us of the fact that in cascade models of turbulence energy dissipates from larger scales down to smaller scales. In our hierarchical model the actual dissipation force at spatial level $k$ is proportional to the velocity difference at the next smaller scale $k+1$ :

$$
\dot{y}^{(k)}=-\Gamma y^{(k+1)}+L^{(k)}(t) \quad k=1, \ldots, N
$$

We now show that this model, with a cascade of size $N$, leads to an initial value problem of the form

$$
\frac{\partial^{n}}{\partial t^{n}} y=(-1)^{N} \Gamma^{N} y+g(t)
$$

where $y(t)=y^{(1)}(t)$ is the velocity difference at the top of the cascade $(k=1), g(t)$ is a driving force at the top of the cascade, and we have assumed periodic boundary conditions at the top and bottom of the cascade, i.e. $y^{(n+1)}(t)=y^{(1)}(t)$.

To derive eq. (2.3), we differentiate eq. (2.2) to obtain

$$
\begin{aligned}
\ddot{y}^{(k)} & =-\Gamma \dot{y}^{(k+1)}+\dot{L}^{(k)}(t) \\
& =+\Gamma^{2} y^{(k+2)}-\Gamma L^{(k+1)}(t)+\dot{L}^{(k)}(t),
\end{aligned}
$$

where in the last step we used eq. (2.2) with $k$ replaced by $k+1$. Further differentiation yields

$$
\frac{\partial^{3}}{\partial t^{3}} y^{(k)}=\Gamma^{2} \dot{y}^{(k+2)}+\ddot{L}^{(k)}(t)-\Gamma \dot{L}^{(k+1)}(t)
$$




$$
=-\Gamma^{3} y^{(k+3)}+\Gamma^{2} L^{(k+2)}(t)-\Gamma \dot{L}^{(k+1)}(t)+\ddot{L}^{(k)}(t) .
$$

Finally, for a cascade with $N$ steps one arrives at

$$
\frac{\partial^{N}}{\partial t^{N}} y^{(k)}(t)=(-1)^{n} \Gamma^{N} y^{(k+N)}+g(t)
$$

where $g(t)$ is a linear combination of derivatives of the driving forces at the various scales:

$$
g(t)=(-\Gamma)^{N-1} L^{(k+N-1)}(t)+(-\Gamma)^{N-2} \dot{L}^{(k+N-2)}+\ldots
$$

Our implemented periodic boundary condition of the cascade $y^{(1)}(t)=y^{(N)}(t)$ simply means that at smallest scales the dynamics should just be the same as at the largest scales, which is a self-similarity assumption. For $N$ odd and defining $y^{(1)}(t)=y(t)$ we arrive at

$$
\frac{\partial^{N}}{\partial t^{N}} y+\Gamma^{N} y=g(t)
$$

which has to be supplemented by a set of $N$ initial conditions, corresponding to initial velocities at the various scales. This initial value problem will be solved in the next section.

\section{The initial value problem}

In the following, we choose as an example $N=7$. In turbulence simulations, the driving forces at various scales of the turbulent flow are only known in numerical form, and given the chaotic nature of the forces it is important to implement high precision numerical methods that optimize the numerical solution of driven velocity fields within a given cascading subdynamics. In the following, we introduce a high-precision non-polynomial spline method for the solution of the initial value problem (IVP) that corresponds to our cascade model.

Consider the following seventh order initial value problem

$$
\left.\begin{array}{ll}
y^{(7)}(t)+f(t) y(t)= & g(t), \quad t \in[a, b], \\
y(a)=u_{0}, & y^{(1)}(a)=u_{1}, \\
y^{(2)}(a)=u_{2}, & y^{(3)}(a)=u_{3}, \\
y^{(4)}(a)=u_{4}, & y^{(5)}(a)=u_{5}, \\
y^{(6)}(a)=u_{6}, &
\end{array}\right\}
$$

where $u_{i} ; i=0,1, \ldots, 6$ are finite real constants while the functions $f(t)$ and $g(t)$ are continuous on $[a, b]$. This is just the dynamics derived in the previous section, provided the effective friction constant $\Gamma$ depends on time $t$. The notation is slightly different since now $y^{(i)}$ denotes the $i$-th derivative with respect to $t$. For our physical application in terms of a cascade-like model, we need to keep the functions $f(t)$ and $g(t)$ quite general since they are unknown in a turbulent flow. Our aim is to provide a proper numerical method to provide a most accurate solution of this IVP. It turns out that a non-polynomial spline method is very useful in this context. For particular choices of the interpolation parameters our method provides optimum results (error term of order five rather than 2), as shown in the following sections. 


\section{Nonpolynomial Spline Method}

To develop the spline approximation to the problem (3.1), the interval $[a, b]$ is divided into $n$ equal subintervals, using the grid points $t_{i}=a+i h ; i=0,1, \ldots, n$, where $h=(b-a) / n$. Consider the following restriction $S_{i}$ of the approximate solution $S$ to each subinterval $\left[t_{i}, t_{i+1}\right], i=0,1, \ldots, n-1$,

$$
\begin{aligned}
S_{i}(t)= & a_{i} \cos \omega\left(t-t_{i}\right)+b_{i} \sin \omega\left(t-t_{i}\right)+c_{i}\left(t-t_{i}\right)^{6}+d_{i}\left(t-t_{i}\right)^{5}+e_{i}\left(t-t_{i}\right)^{4} \\
& +p_{i}\left(t-t_{i}\right)^{3}+q_{i}\left(t-t_{i}\right)^{2}+r_{i}\left(t-t_{i}\right)+v_{i} .
\end{aligned}
$$

Let

$$
\left.\begin{array}{rl}
y_{i}=S_{i}\left(t_{i}\right) & m_{i}=S_{i}^{(1)}\left(t_{i}\right), \\
M_{i}=S_{i}^{(2)}\left(t_{i}\right), & N_{i}=S_{i}^{(4)}\left(t_{i}\right), \\
U_{i}=S_{i}^{(7)}\left(t_{i}\right), &
\end{array}\right\} i=0,1, \ldots, n .
$$

Following [6] and postulating continuous derivatives at knots, consistency relations between the values of splines and their seventh order derivatives at knots are obtained as

$$
\begin{gathered}
\left(\alpha h^{7} U_{i-7}+\beta h^{7} U_{i-6}+\gamma h^{7} U_{i-5}+\delta h^{7} U_{i-4}+\delta h^{7} U_{i-3}+\gamma h^{7} U_{i-2}+\beta h^{7} U_{i-1}+\alpha h^{7} U_{i}\right) \\
=\left[-120 y_{i-7}+840 y_{i-6}-2520 y_{i-5}+4200 y_{i-4}-4200 y_{i-3}+2520 y_{i-2}-840 y_{i-1}+120 y_{i}\right] ; \\
i=7,8, \ldots, n,
\end{gathered}
$$

where

$$
\begin{aligned}
\alpha & =\left(\frac{120(\cos \theta-1)}{\theta^{7} \sin \theta}+\frac{60}{\theta^{5} \sin \theta}-\frac{5}{\theta^{3} \sin \theta}+\frac{1}{6 \theta \sin \theta}\right), \\
\beta & =\left(\frac{600(1-\cos \theta)}{\theta^{7} \sin \theta}-\frac{60(2 \cos \theta-3)}{\theta^{5} \sin \theta}+\frac{5(2 \cos \theta-9)}{\theta^{3} \sin \theta}-\frac{(2 \cos \theta-57)}{6 \theta \sin \theta}\right), \\
\gamma & =\left(\frac{1080(\cos \theta-1)}{\theta^{7} \sin \theta}+\frac{180(2 \cos \theta+1)}{\theta^{5} \sin \theta}+\frac{45(2 \cos \theta+1)}{\theta^{3} \sin \theta}-\frac{(38 \cos \theta-101)}{2 \theta \sin \theta}\right)
\end{aligned}
$$

and

$$
\delta=\left(\frac{600(1-\cos \theta)}{\theta^{7} \sin \theta}-\frac{60(4 \cos \theta+1)}{\theta^{5} \sin \theta}-\frac{5(20 \cos \theta-1)}{\theta^{3} \sin \theta}-\frac{(604 \cos \theta-359)}{6 \theta \sin \theta}\right) .
$$

Here $\theta=\omega h$ is an arbitrary parameter. The relation (4.3) forms a system of $(n-6)$ linear equations in the $(n)$ unknowns $\left(y_{i}, i=1,2, \ldots, n\right)$, while $U_{i}$ is taken from IVP (3.1) to be equal to $-f_{i} y_{i}+g_{i}, i=0,1, \ldots, n$.

Six further equations (end conditions) are required to obtain a complete solution for the $y_{i} s$ appearing in eq. (4.3). These equations are calculated using the method of undetermined coefficients [5] as follows

$$
\begin{gathered}
U_{0}-10 U_{1}+U_{4}=\frac{1}{h^{7}}\left[\frac{512540}{27} y_{0}-20160 y_{1}+1260 y_{2}-\frac{2240}{27} y_{3}+\frac{161000}{9} h y_{0}^{(1)}\right. \\
\left.\quad+\frac{23800}{3} h^{2} y_{0}^{(2)}+\frac{6160}{3} h^{3} y_{0}^{(3)}+280 h^{4} y_{0}^{(4)}\right], \\
U_{1}-\frac{30666}{8867} U_{2}+U_{5}=\frac{1}{h^{7}}\left[\frac{-957600}{8867} y_{1}+\frac{1048320}{8867} y_{2}-\frac{90720}{8867} y_{3}-\frac{866880}{8867} h y_{0}^{(1)}\right.
\end{gathered}
$$




$$
\begin{aligned}
& -\frac{1209600}{8867} h^{2} y_{0}^{(2)}-\frac{829920}{8867} h^{3} y_{0}^{(3)}-\frac{352800}{8867} h^{4} y_{0}^{(4)} \\
& \left.-\frac{87864}{8867} h^{5} y_{0}^{(5)}\right] \\
U_{2}-\frac{278026}{94221} U_{3}+U_{6}=\frac{1}{h^{7}}\left[\frac{-67340}{10469} y_{2}+\frac{80640}{10469} y_{3}-\frac{700}{551} y_{4}-\frac{54040}{10469} h y_{0}^{(1)}\right. & -\frac{4200}{361} h^{2} y_{0}^{(2)}-\frac{20720}{1653} h^{3} y_{0}^{(3)}-\frac{85400}{10469} h^{4} y_{0}^{(4)} \\
U_{3}+U_{7}= & \frac{1}{h^{7}}\left[\frac{10808537040}{487056529} y_{3}-\frac{13373418240}{487056529} y_{4}+\frac{2564881200}{487056529} y_{5}\right. \\
+ & \frac{8243655840}{487056529} h y_{0}^{(1)}+\frac{26287914240}{487056529} h^{2} y_{0}^{(2)} \\
+ & +\frac{40576352880}{487056529} h^{3} y_{0}^{(3)}+\frac{39377200800}{487056529} h^{4} y_{0}^{(4)} \\
& \left.+\frac{25438766892}{487056529} h^{5} y_{0}^{(5)}+\frac{9474762304}{487056529} h^{6} y_{0}^{(6)}\right] \\
& \frac{1}{h^{7}}\left[\frac{2645350155}{436783036} y_{4}-\frac{869117760}{109195759} y_{5}+\frac{831120885}{436783036} y_{6}\right. \\
& +\frac{31279815}{7530742} h y_{0}^{(1)}+\frac{3666455415}{218391518} h^{2} y_{0}^{(2)} \\
& +\frac{3572264955}{109195759} h^{3} y_{0}^{(3)}+\frac{8717751945}{218391518} h^{4} y_{0}^{(4)} \\
+U_{4}+U_{8} & \left.+\frac{3525702999}{109195759} h^{5} y_{0}^{(5)}+\frac{1634628387}{109195759} h^{6} y_{0}^{(6)}\right]
\end{aligned}
$$

and

$$
\begin{aligned}
U_{5}+U_{9}= & \frac{1}{h^{7}}\left[\frac{132838307280}{61865369749} y_{5}-\frac{183300929280}{61865369749} y_{6}+\frac{50462622000}{61865369749} y_{7}\right. \\
& +\frac{82375685280}{61865369749} h y_{0}^{(1)}+\frac{402603647040}{61865369749} h^{2} y_{0}^{(2)} \\
& +\frac{946588828080}{61865369749} h^{3} y_{0}^{(3)}+\frac{1390554453120}{61865369749} h^{4} y_{0}^{(4)} \\
& \left.+\frac{1350858565644}{61865369749} h^{5} y_{0}^{(5)}+\frac{749461929944}{61865369749} h^{6} y_{0}^{(6)}\right] .
\end{aligned}
$$

Basically, one does a power expansion in $h$, and postulates that the low orders in $h$ vanish. Our calculations were done using Mathematica.

The local truncation errors associated with the linear equations (4.4) - (4.9) and (4.3) 
are calculated as

$$
\tilde{t}_{i}= \begin{cases}-5.778 h^{9} y^{(9)}\left(t_{1}\right)+O\left(h^{10}\right), & i=1, \\ -6.472 h^{9} y^{(9)}\left(t_{2}\right)+O\left(h^{10}\right), & i=2, \\ -7.230 h^{9} y^{(9)}\left(t_{3}\right)+O\left(h^{10}\right), & i=3, \\ -19.288 h^{9} y^{(9)}\left(t_{4}\right)+O\left(h^{10}\right), & i=4, \\ -25.620 h^{9} y^{(9)}\left(t_{5}\right)+O\left(h^{10}\right), & i=5, \\ -33.020 h^{9} y^{(9)}\left(t_{6}\right)+O\left(h^{10}\right), & i=6, \\ \frac{1}{2}(-100+25 \alpha+13 \beta+5 \gamma+\delta) h^{9} y^{(9)}\left(t_{i}\right)+O\left(h^{10}\right), & i=7,8, \ldots, n\end{cases}
$$

and

$$
\|\tilde{T}\|=\operatorname{ch}^{9} R=O\left(h^{9}\right), \quad R=\max _{t \in[a, b]}\left|y^{(9)}(t)\right|,
$$

where $c$ is a constant which depends only on the values of $\alpha, \beta, \gamma$ and $\delta$ and is independent of $h$. Moreover, $\alpha, \beta, \gamma$ and $\delta$ are taken such that $\alpha+\beta+\gamma+\delta=60$.

In general, the solution of the system of linear equations (4.4) - (4.9) and (4.3) is second order convergent.

The local truncation error of the system (4.3) can be expressed in the following form

$$
\tilde{t}_{i}=\left\{\begin{array}{l}
2(-60+\alpha+\beta+\gamma+\delta) h^{7} y^{(7)}\left(t_{i}\right)+(-60+\alpha+\beta+\gamma+\delta) h^{8} y^{(8)}\left(t_{i}\right) \\
+\frac{1}{2}(-100+25 \alpha+13 \beta+5 \gamma+\delta) h^{9} y^{(9)}\left(t_{i}\right) \\
+\frac{1}{6}(-120+37 \alpha+19 \beta+7 \gamma+\delta) h^{10} y^{(10)}\left(t_{i}\right) \\
+\frac{1}{24}(-228+337 \alpha+97 \beta+17 \gamma+\delta) h^{11} y^{(11)}\left(t_{i}\right) \\
+\frac{1}{120}(-380+781 \alpha+211 \beta+31 \gamma+\delta) h^{12} y^{(12)}\left(t_{i}\right) \\
+O\left(h^{13}\right), \\
i=7,8, \ldots, n,
\end{array}\right.
$$

therefore, the order of the truncation error $\tilde{t}_{i}$ can be improved to be of order $h^{12}$, if $\alpha=$ $\frac{151}{15}-\frac{\delta}{5}, \beta=-\frac{301}{6}+\delta, \gamma=\frac{1001}{10}-\frac{9 \delta}{5}$. Correspondingly, the end conditions with local truncation error of $O\left(h^{12}\right)$ can be determined as

$$
\begin{aligned}
& U_{0}- \frac{24407}{109} U_{1}-\frac{59362}{109} U_{2}-\frac{10662}{109} U_{3}-\frac{907}{109} U_{4}+U_{5} \\
&=\frac{1}{h^{7}}\left[\frac{66633336}{545} y_{0}-\frac{19958400}{109} y_{1}+\frac{9979200}{109} y_{2}-\frac{4435200}{109} y_{3}+\frac{1247400}{109} y_{4}-\frac{798336}{545} y_{5}\right. \\
&\left.\quad+\frac{9114336}{109} h y_{0}^{(1)}+\frac{1995840}{109} h^{2} y_{0}^{(2)}-\frac{80}{109} h^{7} y_{0}^{(7)}\right], \\
&=\frac{1}{h^{7}}\left[\frac{-4011644165760}{184871023} y_{1}+\frac{8861887188000}{184871023} y_{2}-\frac{73815832428800}{1663839207} y_{3}\right. \\
& \quad+\frac{4618760731200}{184871023} y_{4}-\frac{1470208924800}{184871023} y_{5}+\frac{1826678971040}{1663839207} y_{6} \\
&\left.\quad-\frac{4345911046400}{554613069} h y_{0}^{(1)}-\frac{1035868310400}{184871023} h^{2} y_{0}^{(2)}-\frac{183470425600}{184871023} h^{3} y_{0}^{(3)}\right],
\end{aligned}
$$




$$
\begin{aligned}
& {\left[U_{2}-\frac{13173366154319505819}{604803696004634} U_{3}-\frac{5923535526089565973}{302401848002317} U_{4}\right.} \\
& \left.-\frac{2639790737228529743}{604803696004634} U_{5}+U_{7}\right] \\
& =\frac{1}{h^{7}}\left[\frac{25352931909798309915}{43200264000331} y_{2}-\frac{198185856313975120000}{129600792000993} y_{3}\right. \\
& +\frac{72535593878062755750}{43200264000331} y_{4}-\frac{44672825515677652800}{43200264000331} y_{5} \\
& +\frac{44958164899589796925}{129600792000993} y_{6}-\frac{2139803134054971840}{43200264000331} y_{7} \\
& +\frac{5764036699720950200}{43200264000331} h y_{0}^{(1)}+\frac{7374675959642702700}{43200264000331} h^{2} y_{0}^{(2)} \\
& \left.+\frac{3273172503578299200}{43200264000331} h^{3} y_{0}^{(3)}+\frac{521467194925746900}{43200264000331} h^{4} y_{0}^{(4)}\right] \text {, } \\
& U_{3}-\frac{3169885805313999875741}{17618985121607404312} U_{4}-\frac{544760103861334609083}{17618985121607404312} U_{6}+U_{7} \\
& =\frac{1}{h^{7}}\left[\frac{5366584014500607349360}{19821358261808329851} y_{3}-\frac{1627194491533397967735555}{1127615047782873875968} y_{4}\right. \\
& +\frac{5581229831865256388400}{2202373140200925539} y_{5}-\frac{324347788172453021646845}{158570866094466638808} y_{6} \\
& +\frac{1793040053953186573920}{2202373140200925539} y_{7}-\frac{147257531382013448691645}{1127615047782873875968} y_{8} \\
& -\frac{78317811652764229087465}{845711285837155406976} h y_{0}^{(1)}-\frac{42412130734120984954425}{140951880972859234496} h^{2} y_{0}^{(2)} \\
& -\frac{13509365844145615609375}{35237970243214808624} h^{3} y_{0}^{(3)}-\frac{7826257773953806554675}{35237970243214808624} h^{4} y_{0}^{(4)} \\
& \left.-\frac{447768854035545682017}{8809492560803702156} h^{5} y_{0}^{(5)}\right] \\
& U_{4}-\frac{6169811365491003355386625}{364845537886699795641421} U_{7}+U_{9} \\
& =\frac{1}{h^{7}}\left[\frac{413182203198678792199193360481}{373601830795980590736815104} y_{4}-\frac{1011762526223941981900336800}{364845537886699795641421} y_{5}\right. \\
& +\frac{998387082478934194463004566965}{354629862825872201363461212} y_{6}-\frac{566429213407879867786917120}{364845537886699795641421} y_{7} \\
& +\frac{173230355267937275186019127455}{373601830795980590736815104} y_{8}-\frac{5254626822196644195075230752}{88657465706468050340865303} y_{9} \\
& +\frac{1828802733123508354716945025585}{7565437073618606962420505856} h y_{0}^{(1)} \\
& +\frac{54937023892836663800655898465}{74170951702143205513926528} h^{2} y_{0}^{(2)} \\
& +\frac{319183920456421230207708911935}{315226544734108623434187744} h^{3} y_{0}^{(3)} \\
& +\frac{81879551659139637198985554365}{105075514911369541144729248} h^{4} y_{0}^{(4)} \\
& +\frac{2991554077139003376141526763}{8756292909280795095394104} h^{5} y_{0}^{(5)}
\end{aligned}
$$




$$
\left.+\frac{303485670688565607390252013}{4378146454640397547697052} h^{6} y_{0}^{(6)}\right]
$$

and

$$
\begin{aligned}
& U_{5}+ U_{10} \\
&=\frac{1}{h^{7}}\left[\frac{19038680213948167651954555270266}{43087137994818537402205515625} y_{5}-\frac{3612553213748861716357961962885}{2680364680381671574716400716} y_{6}\right. \\
&+\frac{4872382360659412438888663650}{2757576831668386393741153} y_{7}-\frac{3637650046079073899112436800}{2757576831668386393741153} y_{8} \\
&+\frac{396380487483749310137552942650}{670091170095417893679100179} y_{9} \\
&+\frac{25866940054548307411938055846689}{172348551979274149608822062500} y_{10} \\
&+\frac{38692597856846972600037888063421}{698011635516060305915729353125} h_{0}^{(1)} \\
&+\frac{9138473244848295227157642242648}{46534109034404020394381956875} h^{2} y_{0}^{(2)} \\
&+\frac{2879180812841847947352594547754}{9306821806880804078876391375} h^{3} y_{0}^{(3)} \\
&+\frac{1170428496335992779989052992}{4278998531899220266150065} h^{4} y_{0}^{(4)} \\
&+\frac{2186365487813281315497916274}{15909097105779152271583575} h^{5} y_{0}^{(5)} \\
&\left.+\frac{1319003601532979667100927096}{41363652475025795906117295} h^{6} y_{0}^{(6)}\right] .
\end{aligned}
$$

It turns out that if $\alpha, \beta, \gamma$ and $\delta$ are chosen as $\alpha=\frac{151}{15}-\frac{\delta}{5}, \beta=-\frac{301}{6}+\delta, \gamma=\frac{1001}{10}-\frac{9 \delta}{5}$, then the order of truncation error of eq. (4.3) is $O\left(h^{12}\right)$ and the order of convergence can be improved up to five based on the improved order of the end conditions.

To illustrate the implementation of the method, three examples are discussed in the following section. We choose a driving force at the top of the cascade for which the IVP can be solved analytically, and then investigate the error terms of our numerical method by comparing with the exact solution. 


\section{$5 \quad$ Numerical Examples}

\section{Example 1}

Consider the following initial value problem

$$
\begin{aligned}
y^{(7)}(t)+y(t) & =-\left(t^{2}-43\right) \cos (t)+\left(-1+t^{2}-14 t\right) \sin (t), \quad t \in[-1,1], \\
y(-1) & =0, \\
y^{(1)}(-1) & =2 \sin (1) \\
y^{(2)}(-1) & =-4 \cos (1)-2 \sin (1) \\
y^{(3)}(-1) & =6 \cos (1)-6 \sin (1) \\
y^{(4)}(-1) & =8 \cos (1)+12 \sin (1) \\
y^{(5)}(-1) & =-20 \cos (1)+10 \sin (1) \\
y^{(6)}(-1) & =-12 \cos (1)-30 \sin (1)
\end{aligned}
$$

This basically corresponds to a periodic forcing at the top of the cascade whose amplitude is a particular quadratic function of $t$.

The analytic solution of the above problem is

$$
y(t)=\left(t^{2}-1\right) \sin (t),
$$

meaning the velocity of the driven particle oscillates with increasing amplitude. Since we can solve this example analytically, we can easily determine the numerical error of our method. The observed maximum errors (in absolute values) associated with $y_{i}$, for the problem (5.1), corresponding to the different values of $\alpha, \beta, \gamma$ and $\delta$, are summarized in Table 1.

The observed maximum errors (in absolute values) associated with $y_{i}$, for the problem (5.1),

Table 1: Maximum absolute errors for problem (5.1) in $y_{i}$.

\begin{tabular}{|c|c|c|c|}
\hline$n$ & $\alpha=\frac{1}{2}, \beta=\frac{19}{2}$ & $\alpha=0, \beta=0$ & $\alpha=10, \beta=10$ \\
& $\gamma=\frac{49}{2}, \delta=\frac{51}{2}$ & $\gamma=0, \delta=60$ & $\gamma=10, \delta=30$ \\
\hline 12 & $2.88 \times 10^{-1}$ & $3.04 \times 10^{-1}$ & $2.76 \times 10^{-1}$ \\
\hline 24 & $3.09 \times 10^{-2}$ & $3.56 \times 10^{-2}$ & $2.73 \times 10^{-2}$ \\
\hline 48 & $2.5 \times 10^{-3}$ & $3.9 \times 10^{-3}$ & $1.4 \times 10^{-3}$ \\
\hline 96 & $1.70 \times 10^{-4}$ & $7.37 \times 10^{-4}$ & $3.19 \times 10^{-4}$ \\
\hline
\end{tabular}

corresponding to the improved end conditions are summarized in Table 2. A significant improvement is obtained. 
Table 2: Maximum absolute errors for problem (5.1) in $y_{i}$.

\begin{tabular}{|c|c|}
\hline$n$ & $\left|y\left(t_{i}\right)-y_{i}\right|$ \\
\hline 10 & $2.25 \times 10^{-1}$ \\
\hline 20 & $2.08 \times 10^{-6}$ \\
\hline 40 & $7.50 \times 10^{-7}$ \\
\hline
\end{tabular}

\section{Example 2}

Consider now an exponential forcing at the top of the cascade, namely the following IVP:

$$
\begin{aligned}
& y^{(7)}(t)-y(t)=-7 e^{t}(5+2 t), \quad 0 \leq t \leq 1 \\
& y(0)=0, \quad y^{(1)}(0)=1 \text {, } \\
& y^{(2)}(0)=0, \quad y^{(3)}(0)=-3 \\
& y^{(4)}(0)=-8, \quad y^{(5)}(0)=-15 \text {, } \\
& y^{(6)}(0)=-24 \text {. }
\end{aligned}
$$

The corresponding analytic solution is

$$
y(t)=t(1-t) e^{t} .
$$

In this case, for a limited amount of time, velocity differences grow exponentially, consistent with the chaotic nature of the velocity field in turbulent flows. In this case the observed maximum errors of our method are shown in table 3 .

The observed maximum errors (in absolute values) associated with $y_{i}$, for the problem (5.2), corresponding to the improved end conditions are summarized in Table 4.

\section{Example 3}

Consider the following problem:

$$
\left.\begin{array}{llrl}
y^{(7)}(t) & =7\left(-6 e^{t}+e^{t}(1-t)\right)+\left(-7 e^{t}+e^{t}(1-t)\right) t, & 0 \leq t \leq 1 \\
y(0) & =0, & y^{(1)}(0)=1, & \\
y^{(2)}(0)=0, & y^{(3)}(0)=-3 \\
y^{(4)}(0)=-8, & y^{(5)}(0)=-15, \\
y^{(6)}(0)=-24 . &
\end{array}\right\}
$$

The corresponding analytic solution is again

$$
y(t)=t(1-t) e^{t}
$$


Table 3: Maximum absolute errors for problem (5.2) in $y_{i}$.

\begin{tabular}{|c|c|c|c|}
\hline$n$ & $\alpha=\frac{1}{2}, \beta=\frac{19}{2}$ & $\alpha=0, \beta=0$ & $\alpha=10, \beta=10$ \\
$\gamma=\frac{49}{2}, \delta=\frac{51}{2}$ & $\gamma=0, \delta=60$ & $\gamma=10, \delta=30$ \\
\hline 10 & $1.5 \times 10^{-3}$ & $1.6 \times 10^{-3}$ & $1.5 \times 10^{-3}$ \\
\hline 20 & $1.75 \times 10^{-4}$ & $1.94 \times 10^{-4}$ & $1.60 \times 10^{-4}$ \\
\hline 40 & $1.81 \times 10^{-5}$ & $2.62 \times 10^{-5}$ & $1.32 \times 10^{-5}$ \\
\hline
\end{tabular}

Table 4: Maximum absolute errors for problem (5.2) in $y_{i}$.

\begin{tabular}{|c|c|}
\hline$n$ & $\left|y\left(t_{i}\right)-y_{i}\right|$ \\
\hline 10 & $1.82 \times 10^{-1}$ \\
\hline 12 & $2.15 \times 10^{-8}$ \\
\hline 15 & $3.65 \times 10^{-9}$ \\
\hline
\end{tabular}

Table 5: Maximum absolute errors for problem (5.3) in $y_{i}$.

\begin{tabular}{|c|c|c|c|}
\hline$n$ & $\alpha=\frac{1}{2}, \beta=\frac{19}{2}$ & $\alpha=0, \beta=0$ & $\alpha=10, \beta=10$ \\
& $\gamma=\frac{49}{2}, \delta=\frac{51}{2}$ & $\gamma=0, \delta=60$ & $\gamma=10, \delta=30$ \\
\hline 9 & $2.0 \times 10^{-3}$ & $2.22 \times 10^{-3}$ & $1.5 \times 10^{-3}$ \\
\hline 18 & $2.26 \times 10^{-4}$ & $2.66 \times 10^{-4}$ & $1.60 \times 10^{-4}$ \\
\hline 36 & $2.16 \times 10^{-5}$ & $3.46 \times 10^{-5}$ & $1.32 \times 10^{-5}$ \\
\hline
\end{tabular}


and we get in this case the results shown in table 5 and 6 .

Table 6: Maximum absolute errors for problem (5.3) in $y_{i}$.

\begin{tabular}{|c|c|}
\hline$n$ & $\left|y\left(t_{i}\right)-y_{i}\right|$ \\
\hline 10 & $1.82 \times 10^{-1}$ \\
\hline 12 & $2.33 \times 10^{-8}$ \\
\hline 15 & $1.67 \times 10^{-8}$ \\
\hline
\end{tabular}

\section{Conclusion}

In this paper we showed that hierarchical cascade models, motivated by turbulent flows, can lead to initial value problems of $N$-th order, where $N$ is the number of cascade steps. As an example we considered $N=7$ and designed the optimum strategy to numerically treat the corresponding initial value problem. A non-polynomial spline method was developed for this. The numerical algorithm depends on some parameters $\alpha, \beta, \gamma, \delta$ for which we derived explicit formulas. The method is observed to be second-order convergent for arbitrary choices of the parameters $\alpha, \beta, \gamma$ and $\delta$ such that $\alpha+\beta+\gamma+\delta=60$ but if $\alpha, \beta$, $\gamma$ and $\delta$ are chosen as $\alpha=\frac{151}{15}-\frac{\delta}{5}, \beta=-\frac{301}{6}+\delta, \gamma=\frac{1001}{10}-\frac{9 \delta}{5}$, then the method is of order five, due to the use of improved order end conditions. This is the optimum choice to numerically deal with cascade-like models of this type. In turbulent flows, driving forces and velocity differences often behave chaotically, corresponding to exponential growth for a limited amount of time. Consequently, we tested our method for these and other types of driving forces. We found that the maximum numerical errors observed are indeed very small if improved order end conditions are used.

\section{References}

[1] C. Beck, Phys. Rev E 49, 3641 (1994)

[2] C. Beck, Phys. Rev. Lett. 87, 180601 (2001)

[3] B.L. Sawford, Phys. Fluids A3, 1577 (1991)

[4] N.G. van Kampen, Stochastic Processes in Physics and Chemistry, North Holland, Amsterdam (1981)

[5] Shahid S. Siddiqi and Ghazala Akram, Applied Mathematics Letters, 20, 591 (2007)

[6] Ghazala Akram and Shahid S. Siddiqi, Applied Mathematics and Computation, 181, $708(2006)$ 
[7] Shahid S. Siddiqi and Ghazala Akram, International Journal of Computer Mathematics, 84, 347 (2007)

[8] Shahid S. Siddiqi and Ghazala Akram, Applied Mathematics and Computation, 190, 641 (2007)

[9] Shahid S. Siddiqi and Ghazala Akram, Applied Mathematics and Computation, 199, 559 (2008) 